\title{
Rendimiento a la carcasa de los cuyes alimentados con gramíneas tropicales Axonopus scoparius, Pennisetum SP, Pennisetum purpureum y Tripsacum laxum en Morona Santiago
}

Casing performance of guinea pigs fed with tropical grasses Axonopus scoparius, Pennisetum SP, Pennisetum purpureum and Tripsacum laxum in Morona Santiago

Tamia Noboa Abdo. ${ }^{1}$, Luis Rojas Oviedo. ${ }^{2}$, Luis Condo Plaza. ${ }^{3} \&$ Segundo Shagñay Rea. ${ }^{4}$

\begin{abstract}
.
DOI: https://doi.org/10.33262/concienciadigital.v3i3.1.1386

The performance of male guinea pigs fed with four tropical grasses in Morona Santiago, was developed in the ESPOCH Morona Sede, for which four pastures were used: Axonopus scoparius (Gramalote - T1), Pennisetum sp (Maralfalfa) (T2), Pennisetum purpureum (Elephant grass) (T3) and Tripsacum laxum (Guatemala grass) (T4) with ten repetitions, giving a total of 40 male guinea pigs, the same ones that consumed the indicated pastures from weaning to slaughter (150 days old) and were distributed completely at random and their results were analyzed through variance and Tukey's test ( $\mathrm{p}<0.05$ ). determining that the use of Axonopus scoparius allowed a weaning weight of $247.84 \mathrm{~g}$, the slaughter weight of $1107.52,599.63 \mathrm{~g}$ of carcass weight and its performance without fasting of 54\%, the weight and yield of the viscera, hair and blood was $507.89 \mathrm{~g}$ and $45.70 \%$, the weight and yield only of viscera was $375.83 \mathrm{~g}$ and $33.80 \%$, the weight and yield of the hair was $78.14 \mathrm{~g}$ and $7.06 \%$ and the weight and yield of the blood was $53.92 \mathrm{~g}$ and $4.84 \%$ respectively, indicating that this pasture was more efficient.
\end{abstract}

Key words: Guinea pigs, carcass, Carcass yield, viscera, blood and hair.

\footnotetext{
${ }^{1}$ Escuela Superior Politécnica de Chimborazo, Sede Morona Santiago - Ecuador., tnoboa@espoch.edu.ec

${ }^{2}$ Escuela Superior Politécnica de Chimborazo, Sede Morona Santiago- Ecuador., luis.rojaso@espoch.edu.ec

${ }^{3}$ Universidad Regional Amazónica IKIAM, Miembro de la Red Lechera Latinoamericana lac.plaza@yahoo.com

${ }^{4}$ Escuela Superior Politécnica de Chimborazo, Sede Morona Santiago- Ecuador., segundo.shagnay@espoch.edu.ec
} 


\section{Resumen}

El rendimiento a la carcasa de cuyes machos que fueron alimentados con gramíneas tropicales en Morona Santiago, se realizó en la ESPOCH Sede Morona Santiago, para esto se utilizaron cuatro pastos: Axonopus scoparius (Gramalote - Tratamiento1), Pennisetum sp (Maralfalfa- Tratamiento 2), Pennisetum purpureum (Pasto elefanteTratamiento 3) y Tripsacum laxum (Pasto Guatemala- Tratamiento 4) con diez repeticiones cada uno, dando un total de 40 cuyes machos, los mismos que consumieron los pastos señalados desde el destete hasta el sacrificio (150 días de edad), se distribuyeron completamente al azar y sus resultados se analizaron a través de la varianza y la prueba de Tukey ( $\mathrm{p}<0.05$ ); determinándose que la utilización del Axonopus scoparius permitió un peso al destete de 247,84 g, el peso de la canal al sacrificio de 1107.52, 599.63 $\mathrm{g}$, su rendimiento sin ayuno de $54 \%$, el peso y rendimiento de las vísceras, pelo y sangre fue de $507.89 \mathrm{~g}$ y $45.70 \%$, el peso y rendimiento únicamente de vísceras fue de $375,83 \mathrm{~g}$ y $33.80 \%$, el peso y rendimiento del pelo fue de $78.14 \mathrm{~g}$ y $7.06 \%$ y el peso y rendimiento de la sangre fue de 53.92 g y $4.84 \%$ respectivamente, señalándose que este pasto fue más eficiente.

Palabras claves: Cuyes, carcasa, Rendimiento a la canal, vísceras, sangre y pelo.

\section{Introducción.}

La finalidad de criar animales domésticos es producir carne, leche, huevos, lana, según la especie para satisfacer los requerimientos de la sociedad, partiendo de esta premisa, el propósito de la crianza de cuyes es producir carne, cuya eficiencia se puede medir a partir del rendimiento a la canal y sus desperdicios más conocido como vísceras blancas y rojas a más de la sangre y pelo (Solorzano, J. y Sarria, J. 2014).

La producción de canales de cuy se da, gracias a la utilización de pastos como alimento de esta especie herbívora, en el Cantón Morona se utiliza en alto porcentaje al Axonopus scoparius (Gramalote), como una fuente de alimento en todas las especies herbívoras, a pesar de que su rebrote tardío, el pasto es palatable y posee un alto contenido de agua, de la misma manera en este sector se cultiva Pennisetum sp (Maralfalfa), Pennisetum purpureum (pasto Elefante) y Tripsacum laxum (Guatemala) especies que se utilizan en la alimentación de cuyes (Rojas et al., 2020).

El rendimiento a la canal de los cuyes es prácticamente bajo cuando se faena a los animales considerando las técnicas de seguridad alimentaria como el ayuno respectivo, aunque a nivel artesanal dentro de las familias, estos animales no se someten a este proceso, razón por lo que el rendimiento es completamente diferente, además de considerar el momento óptimo para pesar a la canal que altera indirectamente el rendimiento económico y productivo (Huaman, 2017). 
Existen procesos biológicos que influyen en el crecimiento y desarrollo del Cavia pocellus, que conducen por un lado a cambios fisiológicos, además el músculo esquelético sufre un desarrollo hipertrófico pudiendo alcanzar un rendimiento a la canal de $64 \%$ cuando los animales están en 12 semanas de edad (Rubio et al., Glass, 2005. Vanderlip, 2003, Cruz, 2013 y Meza et al., 2014).

Por otra parte, la carne de cuy posee $76.98 \%$ de humedad, $18.97 \%$ de proteína, $2.65 \%$ de grasa y $1.4 \%$ de cenizas o minerales (Guarniz y Roque, 2019), por lo que se considera una carne de calidad principalmente por el alto contenido de proteína. El cuy pesa aproximadamente $544 \mathrm{~g}$ y el peso de las vísceras de cuyes de tres meses de edad en promedio fue: corazón 2,79 $\pm 0,76 \mathrm{~g}$; pulmones 4,85 $\pm 1,51 \mathrm{~g}$; hígado 23,29 \pm 6,03 g; riñón $6,06 \pm 1,43 \mathrm{~g}$; bazo $1,13 \pm 0,26 \mathrm{~g}$; estómago vacío 5,63 $\pm 1,34 \mathrm{~g}$; estómago lleno $17,33 \pm 7,54 \mathrm{~g} ; \mathrm{y}$ el intestino 85,04 $\pm 14,91 \mathrm{~g}$, dándonos un total de $1401 \mathrm{~g}$, cuando el animal está en ayuno (Chauca el at,. 1997).

\section{Materiales y Métodos.}

El rendimiento de la carcasa de los cuyes alimentados a base de Axonopus scoparius (Gramalote - Tratamiento 1), Pennisetum sp (Maralfalfa - Tratamiento 2), Pennisetum purpureum (pasto Elefante - Tratamiento 3), Tripsacum laxum (Pasto Guatemala Tratamiento 4); se realizó en las instalaciones de la ESPOCH Sede Morona Santiago a una altitud de 1030 m.s.n.m, con una temperatura $24^{\circ} \mathrm{C}$, con la humedad relativa del $80 \%$ y una precipitación media de $1626 \mathrm{~mm}$ anual; para esto se utilizó 40 cuyes machos que fueron distribuidos en cuatro tratamientos con 10 repeticiones cada uno, los mismos que fueron sacrificados luego de 90 días de alimentación con los tratamientos señalados. Las unidades experimentales fueron distribuídas al azar por los cual los resultados experimentales se analizaron bajo un Diseño Completamente al Azar y que su modelo matemático fue: $Y \mathrm{ij}=\mathrm{u}+\mathrm{Tij}+\mathrm{Eij}$.

Siendo: Yij: El valor estimado de la variable, u: La media general, Ti: El efecto de los diferentes tipos de gramíneas tropicales, y Eij: El efecto de la aleatorización de las unidades experimentales en el campo experimental (error experimental), separación de medias se realizó según Tukey, este modelo se realizó con la ayuda del software INFOSTAT versión Estudiantil.

Una vez que los animales concluyeron la fase de engorde bajo el efecto de los diferentes pastos, los animales se dejaron en ayunas por el espacio de 12 horas, para luego pesar y sacrificar mediante el aturdimiento y el respectivo desangrado para garantizar que las canales estén libre de sangre y se pueda dañar, posteriormente a ello, los animales se sumergieron en agua hervida para proceder a sacar el pelo, para luego eviscerar y cuando el animal estuvo libre de las vísceras blancas y rojas, se procedió a dejar una hora en oreo para pesar la canal, se registraron los resultados del pelo y la sangre y fueron sometidos a un proceso estadístico el mismo que se describe y discute en el artículo. 


\section{Resultados y Discusión.}

Al nacimiento, los cuyes criados en el Cantón Morona pesaron entre 99.47 y 128.13 g, los cuales fueron sometidos a diferentes tipos de gramíneas como alimento, y al destete los animales que estaban bajo el efecto del Axonopus scoparius (Gramalote) y Pennisetum $s p$ (Maralfalfa) registraron en promedio 247,84 y 244.98 g, siendo superiores estadísticamente aquellos que fueron alimentados con Pennisetum purpureum (Elefante) y Tripsacum laxum (Guatemala) con los cuales se consiguieron 210.78 y 233.18 g respectivamente.

Los cuyes en ayuno listos para el sacrificio que estaban alimentados con Axonopus scoparius (Gramalote) $1107.52 \mathrm{~g}$, valor que difiere significativamente $(\mathrm{p}<0.01)$ de los tratamientos a base de Pennisetum sp (Maralfalfa), Pennisetum purpureum (Elefante) y Tripsacum laxum (Guatemala) con los cuales se determinó 988.41, 859.19 y 914.14 g respectivamente, determinándose que posiblemente el Axonopus scoparius (Gramalote) es el pasto más digerible en el tracto digestivo del cuy además es más palatable debido a que tiene menor cantidad de vellosidades en comparación con los pastos que propiciaron un menor peso al sacrificio. Hernandez, C. (2015) señala que el peso al sacrificio de los cuyes de engorde a los 3 meses fue de $1023.40 \mathrm{~g}$ similar al del presente trabajo de investigación. Pacuar, (2011) señala que los cuyes engordados a los tres meses de edad con dieta a base de harina de algas obtienen pesos de 1.212,55 g. Tuquinga, (2011) al utilizar una dieta de a base de quinua los cuyes presentaron pesos al sacrificio de 1.107,50 $\mathrm{g}$, y al ser alimentados con levadura de cerveza (Saccharomyces cerevisiae) se obtuvo un peso final de $1.110 \mathrm{~g}$ a los 85 días de edad (Farinango, 2010). Valores superiores a los registrados en el presente trabajo.

El peso a la canal de los cuyes alimentados con Axonopus scoparius (Gramalote) en promedio fue $599.63 \mathrm{~g}$, valor que difiere significativamente $(\mathrm{p}<0.01)$ de los cuyes que recibieron alimentos en base a Pennisetum sp (Maralfalfa), Pennisetum purpureum (Elefante) y Tripsacum laxum (Guatemala) cuyos pesos fueron de 501.34, 459.51 y $483.34 \mathrm{~g}$ respectivamente, aunque se puede mencionar que con la utilización de Gramalote, Maralfalfa, pasto Elefante y Guatemala, el rendimiento a la canal fue de $54.30,50.73,53.55$ y $52.89 \%$ de rendimiento a la canal, valores entre los cuales no registran diferencias significativas ( $\mathrm{p}>0.05$ ), determinándose que este rendimiento no está establecido por el tipo de pasto que se utilice como alimento de los cuyes. Chauca et al., (1997) señala que el peso de la carcasa en cuyes de 3 meses de edad es de $543.77 \mathrm{~g}$ y un rendimiento a la canal de 54.43 a $67.38 \%$ en cuyes machos y Hernández, C. (2015) registra un rendimiento a la canal de $53.97-55.98 \%$, siendo similares a los encontrados en el presente estudio. Mientras que Acosta, A. et al., (2010) al utilizar concentrado en la alimentación de cuyes registro pesos a la canal de $848.17 \mathrm{~g}$ de peso a la canal y con rendimiento a la canal del $73.37 \%$, siendo superiores a los encontrados en el presente trabajo, posiblemente se deba a factores como edad de los cuyes al sacrificio y de los sistemas de alimentación. 
El peso de las vísceras, pelo y sangre de los cuyes alimentados con Gramalote en promedio fue $507.89 \mathrm{~g}$ y al utilizar Maralfalfa fue de $487.07 \mathrm{~g}$, valores que difieren significativamente $(\mathrm{p}<0.01)$ de los cuyes que recibieron alimentos en base a pasto Elefante y Guatemala cuyos pesos fueron de 399.68 y $430.80 \mathrm{~g}$ respectivamente, mientras que el peso porcentual de las vísceras, pelo y sangre al utilizar Gramalote, Maralfalfa, pasto Elefante y Guatemala fue de 45.70, 49.27, 46.45 y $47.11 \%$ de rendimiento, valores entre los cuales no registran diferencias significativas ( $p>0.05)$, determinándose que la proporción de estas vísceras no dependen del tipo de pasto sino posiblemente de la especie de animal doméstico. Coronado (2007) menciona que el $35 \%$ corresponde al peso de las vísceras en cuyes, siendo inferior a los señalado en el presente estudio, mientras que Hernández, C. (2015) señala que el peso de las vísceras es de $46.03 \%$, en la cual están incluidas el pelo y la sangre, valores similares a los obtenidos en el presente estudio.

Únicamente al pesar las vísceras blancas y rojas (intestino grueso, delgado, cuajo, esófago, hígado y pulmones) al utilizar Gramalote y Maralfalfa registraron 375.83 y $367.14 \mathrm{~g}$, valores que difieren significativamente $(\mathrm{p}<0.01)$ del peso de las vísceras de los cuyes alimentados con pasto Elefante y Guatemala con los cuales se obtuvieron pesos de 296.45 y 332.16 g respectivamente, mientras que el mayor porcentaje de vísceras se determinó al utilizar el Maralfalfa y Guatemala registrándose un rendimiento en vísceras de 37.14 y $36.34 \%$, mientras que la utilización de Gramalote y pasto Elefante los pesos de las vísceras fueron de 33.8 y 34.45 respectivamente. Señalándose que, los cuyes alimentados con Gramalote y pasto Elefante no propiciaron el desarrollo de vísceras como se observa al utilizar Maralfalfa y Guatemala. Zamora, S. (2016) señala que el peso de las vísceras en cuyes esta entre 143.00 y $177.50 \mathrm{~g}$, valores que inferiores a los encontrados en el presente estudio, esto posiblemente puede deberse a que en el presente trabajo se utilizó forraje en una alta proporción lo que hizo que en el animal se desarrolle principalmente las vísceras blancas (intestino y estómago). Xicohtencatl, et al., (2013) señalan que el rendimiento de vísceras es del $26.5 \%$, inferior al señalado en el presente trabajo.

Al pesar el pelo de los cuyes alimentados con Gramalote se registró $78.14 \mathrm{~g}$, valor que difiere significativamente $(\mathrm{p}<0.01)$ del peso del pelo de los cuyes alimentados con Maralfalfa, pasto Elefante y Guatemala con los cuales se obtuvieron 73.04, 67.04 y 56.44 g respectivamente, mientras que el mayor porcentaje de pelo se determinó al utilizar pasto Elefante que registró el $7.38 \%$ de pelo, siendo diferente significativamente $(\mathrm{p}<0.01)$ al utilizar Gramalote Maralfalfa y Guatemala con los cuales se obtuvieron 7.06, 7.39 y 6.18 $\%$ respectivamente. Señalándose que en los cuyes alimentados con pasto Elefante se propicia el crecimiento del pelo, no así, al utilizar Gramalote, Maralfalfa y Guatemala. Zamora, S. (2016) señala que el peso del pelo en cuyes a los tres meses es de $43.25 \mathrm{y}$ $51.22 \mathrm{~g}$, valores inferiores a los encontrados en el presente trabajo. Xicohtencatl, et al., (2013) señala que el rendimiento de pelo con relación al peso vivo es de $5.5 \%$, inferior al señalado encontrado en el presente estudio. 
Tabla 1: Rendimiento de los cuyes alimentados con diferentes pastos tropicales Gramalote, Maralfalfa, Pasto Elefante y Pasto Guatemala

\begin{tabular}{|c|c|c|c|c|c|c|c|c|}
\hline \multirow[b]{2}{*}{ Variables } & \multicolumn{8}{|c|}{ Pastos Tropicales } \\
\hline & Gramalote $^{1}$ & & Maralfalfa $^{2}$ & & Elefante $^{3}$ & & Guatemala $^{4}$ & \\
\hline Peso al nacimiento $(\mathrm{g})$ & 126,40 & & 125,71 & & 99,47 & & 128,13 & \\
\hline Peso al destete (g) & 247,84 & $\mathrm{a}$ & 244,98 & $\mathrm{a}$ & 210,78 & $\mathrm{~b}$ & 233,18 & $\mathrm{a}$ \\
\hline Peso al sacrificio (g) & 1107,52 & $\mathrm{a}$ & 988,41 & b & 859,19 & $\mathrm{c}$ & 914,14 & $\mathrm{~b}$ \\
\hline Peso a la canal (g) & 599,63 & a & 501,34 & b & 459,51 & $\mathrm{~b}$ & 483,34 & $\mathrm{~b}$ \\
\hline $\begin{array}{l}\text { Rendimiento a la car } \\
(\%)\end{array}$ & 54,30 & a & 50,73 & $\mathrm{a}$ & 53,55 & $\mathrm{a}$ & 52,89 & $\mathrm{a}$ \\
\hline $\begin{array}{l}\text { Vísceras, pelo y Sang } \\
(\mathrm{g})\end{array}$ & 507,89 & a & 487,07 & a & 399,68 & $\mathrm{c}$ & 430,80 & $\mathrm{~b}$ \\
\hline $\begin{array}{l}\text { Vísceras, pelo y Sang } \\
(\%)\end{array}$ & 45,70 & a & 49,27 & $\mathrm{a}$ & 46,45 & a & 47,11 & $\mathrm{a}$ \\
\hline Vísceras (g) & 375,83 & $\mathrm{a}$ & 367,14 & a & 296,45 & $\mathrm{c}$ & 332,16 & $\mathrm{~b}$ \\
\hline Vísceras (\%) & 33,80 & $\mathrm{c}$ & 37,14 & $\mathrm{a}$ & 34,45 & bc & 36,34 & $a b$ \\
\hline Pelo $(g)$ & 78,14 & $\mathrm{a}$ & 73,04 & b & 67,04 & $\mathrm{c}$ & 56,44 & d \\
\hline Pelo $(\%)$ & 7,06 & $\mathrm{c}$ & 7,39 & $\mathrm{~b}$ & 7,80 & a & 6,18 & $\mathrm{~d}$ \\
\hline Sangre (g) & 53,92 & $\mathrm{a}$ & 46,89 & a & 36,19 & $\mathrm{a}$ & 42,21 & $\mathrm{a}$ \\
\hline Sangre $(\%)$ & 4,84 & $\mathrm{a}$ & 4,74 & $\mathrm{a}$ & 4,21 & $\mathrm{a}$ & 4,59 & $\mathrm{a}$ \\
\hline
\end{tabular}

Letras iguales horizontalmente no difieren significativamente según Tukey ( $>00.05)$.

${ }^{1}$ : Axonopus scoparius; ${ }^{2}$ : Pennisetum $s p ;{ }^{3}$ : Pennisetum purpureum y, ${ }^{4}$ : Tripsacum laxum.

El contenido de sangre en los cuyes alimentados con Gramalote, Maralfalfa, pasto Elefante y Guatemala fue 53.92, 46.89, 36.19 y $42.21 \mathrm{~g}$, de igual manera ocurre con el porcentaje de sangre cuyos reportes fueron $4.84,4.74,4.21$ y $4.59 \%$ respectivamente, valores entre los cuales no difieren significativamente ( $>>0.05$ ). Zamora, S. (2016) señala que el peso de la sangre en cuyes para faenar esta entre 51.33 y $59.33 \mathrm{~g}$, valores que se encuentran dentro del límite superior alcanzado en el presente trabajo. Xicohtencatl, et al., (2013) señala que el rendimiento de la sangre en cuyes es del $3.0 \%$, inferior encontrado en el presente trabajo.

\section{Conclusiones.}

- La utilización del Gramalote permitió obtener un peso en los cuyes al destete de $247.84 \mathrm{~g}$, al sacrificio pesaron $1107.52 \mathrm{~g}$, el peso a la canal fue de $599.63 \mathrm{~g}$, con un rendimiento del $54.30 \%$, las vísceras, pelo y sangre pesaron $507.89 \mathrm{~g}$ que corresponde al $45.70 \%$, únicamente las vísceras pesaron 375.83 g que corresponde al $33.80 \%$, el peso del pelo fue de $78.14 \mathrm{~g}$ equivalente al $7.06 \% \mathrm{y}$ 
la sangre peso 53.92 g equivalente al $4.84 \%$ siendo los indicadores de mayor eficiencia en cuyes.

- Por lo tanto, se recomienda utilizar en la Amazonía pasto Gramalote puesto que permite mejores indicadores de rendimiento del cuy una vez que termina la vida productiva.

\section{Referencias Bibliográficas}

Acosta, A. Díaz, H. Trujillo, J. 2010. Evaluación de tres concentrados comerciales en la etapa de crecimiento-engorde de cuyes. Tesis de Grado. FCP - ESPOCH. Riobamba Ecuador.

Chauca de ZL. 1997. Producción de cuyes (Cavia porcellus) Organización de las Naciones Unidas para la Agricultura y la Alimentación. Roma, Italia. http://www.fao.org/docrep/W6562S/W6562S00.htm

Cruz Mm. 2013. Comportamiento productivo de progenies F2 de cuatro cruzamientos entre grupos raciales de cuyes (Cavia porcellus) de hembras F1 con machos Macabeo y peruano mejorado. Tesis de Ingeniero Agrónomo. Quito: Univ. Central del Ecuador. 80 p.

Coronado Sm. 2007. Manual técnico para la crianza de cuyes en el Valle del Mantaro. Talleres Gráficos PRESSCOM; Huancayo, Perú.

http://es.scribd.com/doc/58472339/2/Propiedades-y-Valor-Nutritivo-de-la-Carne-deCuy.

Farinango, H. 2010. Incidencia de la levadura de cerveza (Saccharomyces cerevisiae) en la fase de recría y engorde del cuy (Cavia porcellus). Imbabura. EC. s.e.p. 1-4

Glass Dj. 2005. Skeletal muscle hypertrophy and atrophy signaling pathways. Int J Biochem Cell Biol 37: 1974- 1984. doi: 10.1016/j.biocel.2005.-04.018.

Guarniz, R. Roque, A. 2019. Efecto del tipo de alimento en el rendimiento d ela carcasa del cuy raza Perú (Cabia porcellus). Universidad Nacional de Trujillo. Facultad de Ciencias Agropecuarias. Escuela profesional de Ingeniería agroindustrial. Tesis para optar por el título de Ingeniero agroindustrial. http://dspace.unitru.edu.pe/bitstream/handle/UNITRU/12710/Guarniz\%20Benites $\% 2 \mathrm{c} \% 20$ Roque $\% 20$ Anselmo.pdf?sequence $=1 \&$ is Allowed $=y$

Hernández, C. 2015. Efecto del sexo y edad de sacrificio sobre los quintos cuartos y la calidad de la canal de cuy. Trabajo de grado previo a la obtención del Título de Ingeniero Agroindustrial. Universidad Nacional de Chimborazo Facultad de Ingeniería carrera de Ingeniería Agroindustrial. Riobamba - Ecuador.

Huaman. D. 2017. Rendimiento carcasa en cuyes (Cavia porcellus) machos raza Perú, alimentados con alfalfa, mixto y concentrado en la estación experimental agraria Chumbibamba-Andahuaylas. Universidad Tecnológica de los Andes filial Andahuaylas facultad de Ingeniería Escuela Profesional de Agronomía. http://repositorio.utea.edu.pe/bitstream/handle/utea/70/Rendimiento\%20carcasa\% 20de\%20cuyes\%20T040_43483459_T.pdf?sequence=1\&isAllowed=y 
Meza Ga, Cabrera Rp, Moran Jj, Meza Ff, Cabrera Ca, Meza Ci, Meza Js, Cabanilla Mg, López Fx, Pincay Jl, Bohórquez T, Ortiz J. 2014. Mejora de engorde de cuyes (cavia porcellus L) a base de gramineas y forrajeras arbustivas tropicales en la zona de Quevedo, Ecuador. Idesia 32(3): 75- 80. doi: 10.4067/S0718-34292014000300010

Paucar. F. 2011. Utilización de Diferentes Niveles de Harina de algas de Agua Dulce en la Alimentación de Cuyes y su Efectos en las Etapas de Gestación- Lactancia, Crecimiento, Engorde. Tesis de grado. Escuela Superior Politécnica de Chimborazo. Escuela de Ingeniería Zootécnica. http://dspace.espoch.edu.ec/bitstream/123456789/1044/1/17T01011.pdf

Rojas, L. Noboa, T. Shagñay, S. \& Condo, L. 2020. Alimentación de cuyes en la fase de crecimiento en base a gramíneas tropicales de Morona Santiago. Revista Conciencia Digital. Vol. 3, N².2, p. 50-59, junio, 2020. ISSN 2600-5859. www.concienciadigital.

Rubio, P. Chávez, J. Febres, G. Y Deza, H. 2018. Predicción de peso de carcasa a la edad de beneficio en cuyes del genotipo Cieneguilla con base a una síntesis de medidas corporales. Revista de Investigaciones Veterinarias del Perú. versión impresa ISSN 1609-9117.

Sánchez, A., Sánchez, S., Godoy, S., Díaz, R. Y Vega, N. 2009. Gramíneas tropicales en el engorde de cuyes mejorados sexados (Cavia porcellus Linnaeus) en la zona de la Maná. Facultad de Ciencias Pecuarias, Universidad Técnica Estatal de Quevedo. Mocache, Los Ríos, Ecuador. Publicado en Ciencia y Tecnología 2: pp. 25-28. 2009. Disponible en http://dialnet.unirioja.es/servlet/articulo?codigo=4053241

Solorzano, A. Y Sarria, J. 2014. Crianza, producción y comercialización de cuyes. Edit. Macro. Lima - Perú. pp 190.

http://www.solucionespracticas.org.pe/fichastecnicas/pdf/Crianza\%20de\%20cuyes.pdf

Tuquinga, F. (2011). Evaluación de Diferentes Niveles de Desecho de Quinua en la Etapa de Crecimiento y Engorde de Cuyes. Tesis de Grado. Escuela superior Politécnica de Chimborazo Facultad de Ingeniería Zootécnica

Vanderlip S. 2003. The guinea pig handbook. New York, USA: Barrons. 161 p.

Xicohtencatl-Sánchez Pascual G, Barrera-Zúñiga Samuel, Tiodolo Orozco-Orozco, Torres-Sandoval Sigfredo Fidel Mar, Monsivais-Isiordia Roberto. 2013. Parámetros productivos de cuyes (Cavia porcellus) del nacimiento al sacrificio en Nayarit, México. Abanico Veterinario.

Zamora, S. 2016. Rendimiento y composición corporal de cuyes (Cavia porcellus) suplementados con tres niveles de harina de sangre bovino (bos taurus) procesada artesanalmente. Tesis para obtener el grado académico de maestro en ciencias con mención en Producción y Sanidad Animal. Universidad Nacional de Trujillo Escuela de Postgrado sección de postgrado en Ciencias Agropecuarias.

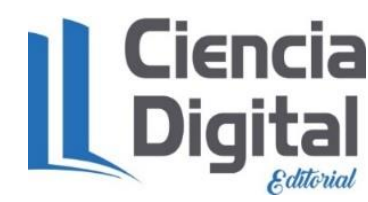




\section{PARA CITAR EL ARTÍCULO INDEXADO.}

Noboa Abdo, T., Rojas Oviedo, L., Condo Plaza, L., \& Shagñay Rea, S. S. R. (2020). Rendimiento a la carcasa de los cuyes alimentados con gramíneas tropicales Axonopus scoparius, Pennisetum SP, Pennisetum purpureum y Tripsacum laxum en Morona Santiago. $\quad$ ConcienciaDigital, $\quad 3(3.1), \quad 243-251$. https://doi.org/10.33262/concienciadigital.v3i3.1.1386

\section{Liencia}

El artículo que se publica es de exclusiva responsabilidad de los autores y no necesariamente reflejan el pensamiento de la Revista Conciencia Digital.

El artículo queda en propiedad de la revista y, por tanto, su publicación parcial y/o total en otro medio tiene que ser autorizado por el director de la Revista Conciencia Digital.

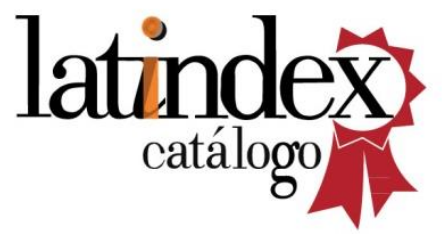

